\title{
Plasma intestinal alkaline phosphatase and intermediate molecular mass gamma glutamyltransferase activities in the differential diagnosis of jaundice
}

\author{
D COLlins, M F GOOLD, S B ROSALKI, P D MAYNE, A Y FOO
}

From the Department of Chemical Pathology and Human Metabolism, The Royal Free Hospital and School of Medicine, London

SUMMARY An assessment was made of the value of: (i) the demonstration of intestinal alkaline phosphatase in plasma for the differentiation of intrahepatic from post-hepatic jaundice in 122 jaundiced patients; and (ii) the demonstration of an intermediate molecular mass gamma glu- $N$ tamyltransferase in plasma for the identification of post-hepatic cholestasis in 74 jaundiced patients. 은 The first test had a diagnostic sensitivity of only $32 \%$ with a specificity of $86 \%$; the second test had a sensitivity of $50 \%$ and specificity of $75 \%$. It is concluded that neither procedure is worth while.

The plasma activities of alkaline phosphatase (ALP; EC 3.1.3.1) and gamma glutamyltransferase (GGT; EC 2.3.2.2) are very much increased in cholestatic liver disease, but differention between intrahepatic and post-hepatic jaundice is not possible by their measurement alone. It has, however, been suggested that the demonstration in plasma by electrophoresis of the intestinal isoenzyme of ALP or of an intermediate molecular mass form of GGT can facilitate differentiation. Intestinal ALP was reported in almost $50 \%$ of patients with intrahepatic jaundice but was absent in post-hepatic disease ${ }^{1}$; the GGT isoform was found to be prominent in about $90 \%$ of patients with post-hepatic jaundice compared with less than $5 \%$ of those with intrahepatic obstruction. ${ }^{2}$ To assess the values of these determinations we measured both isoenzymes in patients with cholestasis. In an initial study we examined intestinal ALP by polyacrylamide gel electrophoresis, as originally recommended, ${ }^{1}$ and by electrophoresis on cellulose acetate membrane which is more convenient for the demonstration of ALP. Cellulose acetate electrophoresis proved the more sensitive procedure and was used alone in a further more extensive study of intestinal ALP. The latter study was also used to evaluate the diagnostic value of the GGT isoforms.

\section{Material and methods}

INITIAL STUDY

Forty seven jaundiced patients, all with increase plasma ALP activity and total bilirubin concentrations were studied: 33 had intrahepatic and 14 post-hepatic jaundice. Diagnosis was based on clinical findings, together with liver function and serological tests, supplemented by ultrasound examination, radiology, liver histology or laparotomy (table 1).

\section{ADDITIONAL STUDY}

Seventy five patients with cholestasis and increased plasma ALP and GGT activity were studied: 45 had intrahepatic and 30 post-hepatic jaundice. Diagnosis was established as above (table 1).

\section{LABORATORY STUDIES}

Plasma total ALP and GGT activities and total bilirubin concentration were measured by Technicon SMAC methods. For isoenzyme studies, plasma sample were stored at $-18^{\circ} \mathrm{C}$ and examined within one month of collection. Alkaline phosphatase iso- N enzymes were separated by both cellulose acetate ${ }^{3}$ 음 and polyacrylamide gel electrophoresis ${ }^{4}$ for the initial 0

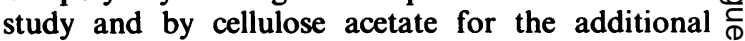
study. In both procedures ALP isoenzymes were $\stackrel{\oplus}{+}$ shown using the chromogenic substrate, 5-bromo-4- 7 chloro-3-indolylphosphate, p-toluidine salt. The pres- $\overrightarrow{\bar{\Phi}}$ 
Table 1 Clinical diagnosis in jaundiced patients studied

\begin{tabular}{llll}
\hline & & $\begin{array}{c}\text { Initial study } \\
(n=33)\end{array}$ & $\begin{array}{l}\text { Additional study } \\
(n=45)\end{array}$ \\
\hline $\begin{array}{lll}\text { Intrahepatic jaundice } \\
\text { Primary biliary cirrhosis }\end{array}$ & 16 & 15 \\
Cirrhosis: & Alcoholic & 4 & 2 \\
Hepatitis: & Wilson's & 1 & -1 \\
& Chronic active & 3 & 4 \\
Viral & 1 & 2 \\
Drug & Alcoholic & -11 \\
Carcinoma: & Primary & 3 & 4 \\
Infiltration & Secondary & 3 & 4 \\
Cyst & & 1 & 1 \\
& & 1 & 1 \\
Post-hepatic jaundice & Initial study & Additional study \\
Gallstone & & $(n=14)$ & $(n=30)$ \\
Benign stricture & 6 & 6 \\
Carcinoma: & Pancreas & 2 & 6 \\
& Gall bladder & 3 & 9 \\
& Bile ducts & 2 & 2 \\
& & & 7 \\
\hline
\end{tabular}

chloro-3-indolylphosphate, $\mathrm{p}$-toluidine salt. The presence of intestinal ALP migrating in the $\beta$ globulin position was especially noted. The detection limit for intestinal ALP activity is $5 \mathrm{U} / 1$ at $37^{\circ} \mathrm{C}$.

For the additional study, intestinal ALP was also quantified from activity remaining after inhibition with p-bromotetramisole. ${ }^{56}$ Blood groups on these patients were also determined.

Gamma glutamyltransferase was separated by gradient polyacrylamide gel electrophoresis ${ }^{7}$ and multiple molecular forms were shown by staining with gamma-glutamyl- $\alpha$-naphthylamide substrate followed by diazonium coupling with fast blue B. ${ }^{8}$ The presence of the intermediate molecular mass form, designated by Wenham, Horn, and Smith $^{9}$ as band IIB, migrating as a compact band about halfway between the origin and albumin, was especially noted and, when present, its relative activity visually compared with the more diffuse and more cathodal band IIA. Differences in activity between the two fractions were always clear cut and unequivocal. The detection limit for GGT activity using this method is less than $15 \mathrm{U} / 1$ at $37^{\circ} \mathrm{C}$.

\section{Results}

\section{INITIAL STUDY}

Intestinal ALP was present in $14(42 \%)$ of the 33 patients with intrahepatic jaundice by cellulose acetate electrophoresis and in nine $(27 \%)$ of these patients by polyacrylamide gel electrophoresis. Intestinal ALP was observed in one (7\%) of the 14 patients with post-hepatic jaundice by both electrophoretic methods. There was a significant difference in the prevalence of intestinal ALP between the two groups of patients by the $\chi^{2}$ test $(p<0.02)$ for cellulose ace-
Table 2 Diagnostic value of intestinal ALP in distinguishing intrahepatic $(n=33)$ from post-hepatic jaundice $(n=14)$ (initial study)

Cellulose acetate (No/\%) Polyacrylamide gel (No/\%)

\begin{tabular}{lll}
\hline Sensitivity & $(14 / 33) 42$ & $(9 / 33) 27$ \\
Specificity & $(13 / 14) 93$ & $(13 / 14) 93$ \\
PV + & $(14 / 15) 93$ & $(9 / 10) 90$ \\
PV - & $(13 / 32) 41$ & $(13 / 37) 35$ \\
Efficiency & $(27 / 47) 57$ & $(22 / 47) 47$ \\
\hline
\end{tabular}

PV $=$ predictive value.

tate electrophoresis, but not for polyacrylamide gel. Table 2 shows the diagnostic value of intestinal ALP in distinguishing intrahepatic from post-hepatic jaundice.

\section{ADDITIONAL STUDY}

\section{$A L P$ isoenzymes}

Intestinal ALP was detected in $11(24 \%)$ of the 45 patients with intrahepatic jaundice and in five (17\%) of the 30 with post-hepatic jaundice. There was no significant difference in the prevalence of intestinal ALP between intrahepatic and post-hepatic jaundiced patients by $\chi^{2}$ testing.

Table 3 shows the diagnostic value of intestinal ALP for intrahepatic jaundice in all patients and in patients of blood groups $\mathrm{O}$ and $\mathrm{A}$ considered separately. The number of patients in blood groups $B$ and AB was too small to be included for statistical analysis.

The figure shows the activity of intestinal ALP in patients of blood groups $\mathrm{O}$ and $\mathrm{A}$ and cholestasis. There was no significant difference in intestinal ALP activity between intrahepatic jaundice patients with blood group $\mathrm{O}(\mathrm{n}=20$, median $5.5 \mathrm{U} / \mathrm{l}$, range $0-54$ $\mathrm{U} / \mathrm{l})$ and post-hepatic jaundice patients with blood group $O(n=12$, median $3 \mathrm{U} / \mathrm{l}$, range $0-32 \mathrm{U} / \mathrm{l})$ or intrahepatic jaundice patients with blood group $\mathrm{A}$ ( $\mathrm{n}=17$, median $4 \mathrm{U} / \mathrm{l}$, range $0-8 \mathrm{U} / \mathrm{l}$ ) and posthepatic jaundice patients with blood group $O(n=9$, median $0 \mathrm{U} / 1$, range $0-9 \mathrm{U} / \mathrm{l}$ ) by Mann-Whitney $\mathrm{U}$ testing.

Table 4 shows the diagnostic value of intestinal ALP, in both the initial and additional studies combined.

Table 3 Diagnostic value of intestinal ALP in distinguishing intrahepatic $(n=45)$ from post-hepatic jaundice $(n=30)$ (additional study)

\begin{tabular}{lllll}
\hline & Overall $(N o / \%)$ & Group $O(N o / \%)$ & Group $A(N o / \%)$ \\
\hline Sensitivity & $(11 / 45) 24$ & $(10 / 21) 48$ & $(0 / 20)$ & 0 \\
Specificity & $(25 / 30) 83$ & $(10 / 14) 71$ & $(10 / 10)$ & 100 \\
PV+ & $(11 / 16) 69$ & $(10 / 14) 71$ & $(0 / 0)$ & -1 \\
PV - & $(25 / 59) 42$ & $(10 / 21) 48$ & $(10 / 30)$ & 33 \\
Efficiency & $(36 / 75) 48$ & $(20 / 35) 57$ & $(10 / 30)$ & 33 \\
\hline
\end{tabular}

PV $=$ predictive value. 

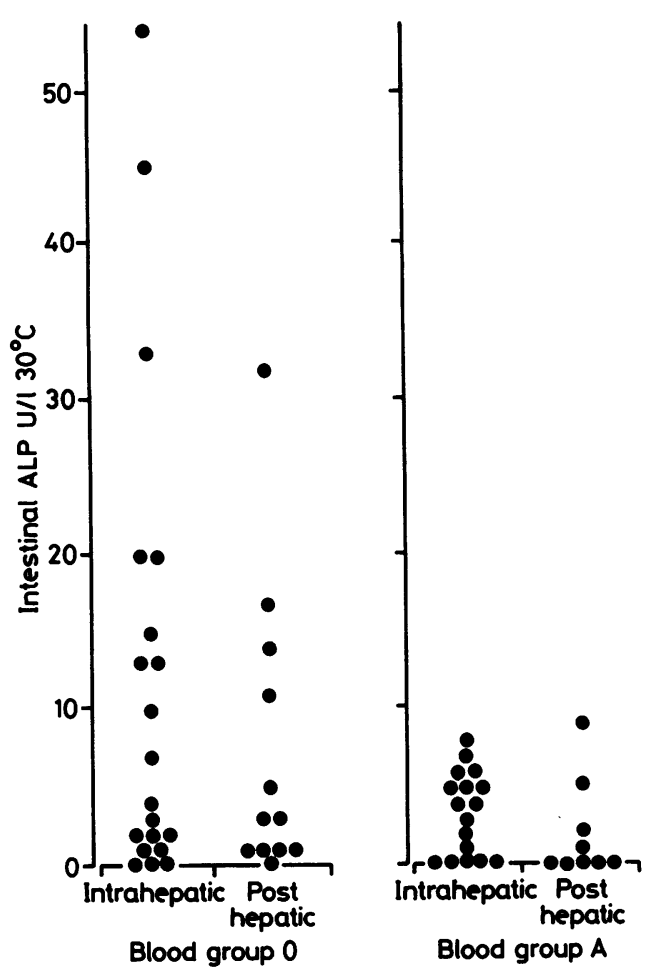

Figure Blood group status and intestinal ALP activity in plasma of patients with jaundice.

\section{GGT molecular forms}

On polyacrylamide gradient gel, up to four GGT bands were observed, numbered from the anode as GGT IV, GGT IIB, GGT IIA and GGT I, the last remaining at the origin. In the 30 patients with posthepatic jaundice $15(50 \%)$ had GGT IIB activity in the absence of or at greater activity than IIA, compared with $11(25 \%)$ of 44 with intrahepatic jaundice (serum from one patient with intrahepatic jaundice was insufficient in volume for this examination). The difference was significant by the $\chi^{2}$ test $(\mathrm{p}<0.05)$. As

Table 4 Diagnostic value of intestinal ALP in distinguishing intrahepatic $(n=78)$ from post-hepatic jaundice $(n=44)$ (initial and additional study combined)

\begin{tabular}{ll}
\hline & Intestinal $(\mathrm{No} / \%)$ \\
\hline Sensitivity & $(25 / 78) 32$ \\
Specificity & $(38 / 44) 86$ \\
PV+ & $(25 / 31) 81$ \\
PV - & $(38 / 91) 42$ \\
Efficiency & $(63 / 122) 52$ \\
\hline
\end{tabular}

$\mathrm{PV}=$ predictive value. a test for post-hepatic jaundice, however, GGT IIB activity in the absence of or at greater activity than IIA had a test sensitivity of only $50 \%$ (15 of 30 ) with a specificity of $75 \%$ (33 of 44$)$, a predictive value of a positive test $\left(\mathrm{PV}^{+}\right)$of $58 \%(15$ of 26$)$ and of a negative test $\left(\mathrm{PV}^{-}\right)$of $69 \%$ (33 of 48$)$, and an efficiency of $65 \%$ (48 of 74).

\section{Discussion}

Intestinal ALP is increased in plasma in intrahepatic disease, particularly cirrhosis, and is unusual in posthepatic cholestasis. ${ }^{10-12}$ Numerous studies have also confirmed that both in health ${ }^{1314}$ and in liver disease $^{1215}$ the prevalence and activity of this isoenzyme is related to blood group, being more pronounced in subjects of blood group $\mathrm{O}$ and $\mathrm{B}$ compared with those of blood group A.

Warnes, Hine, and Kay ${ }^{1}$ suggested that the use of electrophoresis to show intestinal ALP in plasma could differentiate intrahepatic from post-hepatic jaundice. They found that this isoenzyme was present in $45 \%$ of 53 patients with intrahepatic disease and absent in 15 patients with post-hepatic lesions. They claimed that when a raised serum ALP is found in a patient with jaundice and an intestinal band is present on electrophoresis, the lesion is likely to be intrahepatic. It was suggested that post-hepatic biliary obstruction interfered with the absorption of intestinal ALP, whereas hepatocyte damage might reduce its plasma clearance. ${ }^{16}$ Two deficiencies of the study: were recognised by its authors: the rarity of the intestinal band in some varieties of intrahepatic jaundice of non-cirrhotic origin, and a failure to take into account the blood group distribution in the two jaundiced groups studied.

The initial study that we carried out attempted to repeat Warnes's work using polyacrylamide electrophoresis, as originally recommended, and cellulose acetate electrophoresis, which we have found more convenient and generally more sensitive. We did indeed confirm the original suggestions that when the intestinal band was present in the jaundiced patient it was likely to be intrahepatic. Sensitivity, however, was poor $(42 \%$ with cellulose acetate and lower still at $27 \%$ with polyacrylamide gel), specificity was not absolute, and overall efficiency (accuracy) was poor $(57 \%)$. When this study was extended to encompass additional subjects, even this level of performance was not maintained, with a sensitivity of only $32 \%$ in all 78 cases of intrahepatic disease studied using cellulose acetate and a specificity of $86 \%$ in the 44 cases of post-hepatic jaundice. The likelihood of an intestinal band indicating intrahepatic disease $(\mathrm{PV}+)$ was only $81 \%$. Thus in less than one third of the patients with intrahepatic jaundice was the test positive, and almost 
one fifth of positive tests yielded an erroneous diagnosis.

When blood group was taken into account, test performance was even less helpful, sensitivity was reduced to zero in the 20 patients of blood group A with intrahepatic disease, and specificity reduced to $71 \%$ in the 14 patients of blood group $O$ with a post-hepatic lesion. Quantitative determination of intestinal ALP activity by bromotetramisole inhibition confirmed the validity of our electrophoretic studies, with occasional high concentrations of intestinal ALP in patients with intrahepatic jaundice (especially of blood group A) and considerable overlap of activity with post-hepatic disease.

The finding by Wenham, Horn, and Smith ${ }^{9}$ that a specific GGT fraction of intermediate molecular mass (IIB: $250-500 \mathrm{kDa}$ ) shown by electrophoresis was present in plasma in large amounts only in patients with post-hepatic jaundice raised hopes yet again of a useful diagnostic test for the differentiation of intrahepatic and post-hepatic jaundice. In a study of 16 patients with post-hepatic and 48 patients with intrahepatic biliary obstruction these authors ${ }^{2}$ found the presence of band IIB in the absence of or at activity greater than band IIA to be indicative of post-hepatic jaundice, with a sensitivity and specificity of about $90 \%$.

Although providing general support for a greater prevalence of the GGT IIB fraction in post-hepatic disease, our studies did not confirm a useful diagnostic role for the determination. Test sensitivity was only $50 \%$ and specificity only $75 \%$ in the 74 cases we examined.

Therefore, while we would agree that intestinal ALP is more often demonstrable in intrahepatic jaundice and that GGT IIB is more common in post hepatic disease, we conclude that their low sensitivity and limited specificity, and the labour required for their determination does not represent a worthwhile undertaking for the differential diagnosis of jaundice using these isoenzyme fractions.

We thank Professor N McIntyre, Academic Department of Medicine, for permission to study his patients.

\section{References}

1 Warnes TW, Hine P, Kay G. Intestinal alkaline phosphatase in the diagnosis of liver disease. Gut 1977;18:274-8.

2 Wenham DR, Horn DB, Smith AF. Multiple forms of $\gamma$-glutamyltransferase: a clinical study. Clin Chem 1985;31: 559-72.

3 Anido G, Soto A, McBeth CH, Romero P. Alkaline phosphatase isoenzyme in diagnosis. Quad Sclavo Diagn 1972;8:541-2.

4 Smith I, Lighstone PJ, Perry JD. Separation of human tissue alkaline phosphatases by electrophoresis on acrylamide disc gel. Clin Chim Acta 1968;19:499-515.

5 Van Belle H, De Broe ME, Wieme RJ. L-p-bromotetramisole, a new reagent for use in measuring placental or intestinal alkaline phosphatase in human serum. Clin Chem 1977;23:454-9.

6 Doellgast GJ, Meis PJ. Use of specific inhibitors to discriminate alkaline phosphatase isoenzymes originating from human liver, placenta and intestine: absence of meconial alkaline phosphatase in maternal serum. Clin Chem 1979;25:1230-3.

7 Wenham PR, Horn DB, Smith AF. The nature of $\gamma$-glutamyltransferase and other hepatocyte plasma membrane enzymes in human bile. Clin Chim Acta 1982;124:303-13.

8 Wenham PR, Price CP, Sammons HG. A short review of techniques for the localisation of $\gamma$-glutamyltransferase isoenzymes after electrophoresis. Ann Clin Biochem 1978;15:146-50.

9 Wenham PR, Horn DB, Smith AF. Physical properties of $\gamma$-glutamyltransferase, alkaline phosphatase and leucine aminopeptidase in the serum of patients affected by various liver disease. Clin Chim Acta 1984;141:215-8.

10 Fishman WH, Inglis NI, Krant MJ. Serum alkaline phosphatase of intestinal origin in patients with cancer and with cirrhosis of the liver. Clin Chim Acta 1965;12:298-313.

11 Newton MA. A distinctive fraction of alkaline phosphatase in health and disease. J Clin Pathol 1966;19:491-5.

12 Barakat M, Grubb MN, Goyal RK, Hersh T. Intestinal alkaline phosphatase in patients with liver disease. Dig Dis 1971;16:1102-6.

13 Arfors KE, Beckman L, Lundin LD. Genetic variation of human serum phosphatases. Acta Genet 1963;13:89-94.

14 Bamford KF, Harris H, Luffman JE, Robson EB, Cleghorn TH. Serum alkaline phosphatase and the ABO blood groups. Lancet 1965;i:530-1.

15 Stolbach LL, Krant MJ, Inglis NI, Fishman WH. Correlation of serum L-phenylalanine-sensitive alkaline phosphatase, derived from intestine, with the ABO blood group of cirrhotics. Gastroenterology 1967;52:819-27.

16 Warnes TW, Hine P, Kay G, Smith A. Intestine alkaline phosphatase in bile: evidence for an enterohepatic circulation. Gut 1981;22:493-8.

Requests for reprints to: Dr S B Rosalki, Department of Chemical Pathology and Human Metabolism, The Royal Free Hospital, Pond Street, Hampstead, London NW3 2QG, England. 